University of Nebraska - Lincoln

DigitalCommons@University of Nebraska - Lincoln

\title{
Seasonal Malaria Attack Rates in Infants and Young Children in Northern Ghana
}

\author{
J. Kevin Baird \\ U.S. Naval Medical Research Unit \#2, jkevinbaird@yahoo.com \\ S. Owusu Agyei \\ Navrongo Health Research Centre \\ Gregory C. Utz \\ U.S. Naval Medical Research Unit \#3 \\ Kwadwo Koram \\ Noguchi Memorial Institute for Medical Research \\ Mazie J. Barcus \\ U.S. Naval Medical Research Unit \#2 \\ See next page for additional authors
}

Follow this and additional works at: https://digitalcommons.unl.edu/usnavyresearch

Baird, J. Kevin; Agyei, S. Owusu; Utz, Gregory C.; Koram, Kwadwo; Barcus, Mazie J.; Jones, Trevor R.; Fryauff, David J.; Binka, Fred N.; Hoffman, Stephen L.; and Nkrumah, Francis N., "Seasonal Malaria Attack Rates in Infants and Young Children in Northern Ghana" (2002). U.S. Navy Research. 54.

https://digitalcommons.unl.edu/usnavyresearch/54

This Article is brought to you for free and open access by the U.S. Department of Defense at DigitalCommons@University of Nebraska - Lincoln. It has been accepted for inclusion in U.S. Navy Research by an authorized administrator of DigitalCommons@University of Nebraska - Lincoln. 


\section{Authors}

J. Kevin Baird, S. Owusu Agyei, Gregory C. Utz, Kwadwo Koram, Mazie J. Barcus, Trevor R. Jones, David J. Fryauff, Fred N. Binka, Stephen L. Hoffman, and Francis N. Nkrumah 


\title{
SEASONAL MALARIA ATTACK RATES IN INFANTS AND YOUNG CHILDREN IN NORTHERN GHANA
}

\author{
J. KEVIN BAIRD, S. OWUSU AGYEI, GREGORY C. UTZ, KWADWO KORAM, MAZIE J. BARCUS, TREVOR R. JONES, \\ DAVID J. FRYAUFF, FRED N. BINKA, STEPHEN L. HOFFMAN, AND FRANCIS N. NKRUMAH \\ Naval Medical Research Center, Silver Spring, Maryland; Navrongo Health Research Centre, Navrongo, Ghana; U.S. Naval Medical \\ Research Unit No. 3, Cairo, Egypt; Noguchi Memorial Institute for Medical Research, Accra, Ghana
}

\begin{abstract}
The incidence density of infection and disease caused by Plasmodium falciparum in children aged six to 24 months living in the holoendemic Sahel of northern Ghana was measured during the wet and dry seasons of 1996 and 1997. At the beginning of each season, a cohort composed of 259 and 277 randomly selected children received supervised curative therapy with quinine and Fansidar ${ }^{\circledR}$ and primaquine for those with normal glucose-6-phosphate dehydrogenase activity. The 20 weeks of post-therapy follow-up consisted of three home visits weekly and examination of Giemsastained blood films once every two weeks. Blood films were also taken from children brought to clinic with illness. The incidence density of parasitemia after radical cure was 4.7 infections/person-year during the dry season and 7.1 during the wet season (relative risk $=1.51,95 \%$ confidence interval $[\mathrm{CI}]=1.25-1.81 ; P=0.00001$ ). Although the mean parasitemia count at time of reinfection in the dry season $(3,310 / \mu l)$ roughly equaled that in the wet season $(3,056 / \mu \mathrm{l} ; P$ $=0.737)$, the risk ratio for parasitemia $>20,000 / \mu$ l during the wet season was $1.71(95 \% \mathrm{CI}=1.2-2.4 ; P=0.0025)$. The risk ratio for parasitemia $>20,000 / \mu \mathrm{l}$ with fever during the wet season was $2.45(95 \% \mathrm{CI}=1.5-4.1 ; P=0.0002)$. The risk ratio for anemia (hemoglobin $<8 \mathrm{~g} / \mathrm{dl}$ ) at first post-radical cure parasitemia showed no difference between seasons $(1.0 ; 95 \%$ CI $=0.73-1.4 ; P=0.9915)$. We did not see seasonal differences in anemia known to exist in this region, probably because the longitudinal cohort design using first parasitemia as an end point prevented the subjects from developing the repeated or chronic infections required for anemia induction. These findings bear upon the design of malaria drug and vaccine trials in holoendemic areas.
\end{abstract}

\section{INTRODUCTION}

The risk of infection by Plasmodium falciparum within endemic regions varies widely across geographic and seasonal parameters. If one gauges risk as the time to reach a proportion of 1.0 (i.e., $100 \%$ of those at risk have been infected), holoendemic areas of sub-Saharan Africa often yield estimates of just $8-12$ weeks. ${ }^{1-3}$ This reflects an incidence density of approximately five infections/person-year. In contrast, the time to $100 \%$ risk in most of the endemic regions of the Amazon basin ranges between five and 10 years, or an incidence density of approximately 0.1 infections/person-year. ${ }^{4}$

Risk of infection has been linked to patterns of morbidity and mortality caused by $P$. falciparum. ${ }^{5-9}$ Severe anemia represents the primary cause of malaria-related death where the highest risk of infection prevails, while death due to cerebral malaria predominates in areas where infection risk is lower. Naturally acquired immunity in older children and adults in holoendemic Africa effectively prevents either outcome in those groups, but degree of exposure in infants and small children defines risk of specific disease outcomes. Protection from cerebral malaria requires the relatively heavy, uninterrupted exposure such as occurs through the Sahel of subSaharan Africa, but this carries risk of severe anemia.

The threshold of exposure for protection from cerebral malaria versus that for significant risk of severe anemia is not known. The argument has been made that diminishing risk of severe anemia by reducing exposure to infection, e.g., with mosquito bed nets, may increase susceptibility to cerebral malaria. ${ }^{6}$ However, the basis of that argument lies in empirical observations. It remains possible that an exposure level ideal for maintaining protection against cerebral malaria lies well below the exposure of risk for severe anemia. This represents a critical question in the context of control strategies intended to diminish disease and death caused by malaria, especially in sub-Saharan Africa. Few studies in the region have linked disease risks to quantitatively measured incidence density of infection. Thus, it is difficult to begin to assign optimal exposure thresholds, i.e., incidence densities that induce protective immunity from cerebral malaria but below those leading to severe anemia.

We measured the incidence density of infection by $P$. falciparum in infants and young children residing in holoendemic northern Ghana, where seasonal severe anemia is the major cause of malaria-related morbidity. ${ }^{10}$ Incidence density was measured as the post-radical cure attack rate for $P$. falciparum parasitemia. This work provides relatively precise measures of incidence that may be linked to established patterns of disease risk in the region, i.e., high risk of severe anemia among infants and young children. These studies provide a foundation for planning intervention trials in the region, and contribute to the process of assigning risk to defined degrees of exposure to infection.

\section{MATERIALS AND METHODS}

Study site. The Kassena-Nankana District in the Upper East Region of northern Ghana in western Africa (Figure 1) served as the study site. Approximately 140,000 people reside in this district of typical Guinea savanna with sharply distinct wet and dry seasons (Figure 2). Most residents practice subsistence farming, predominantly raising livestock and growing millet. They live within traditional compounds of extended family groups. An on-going census of the population conducted by the Navrongo Health Research Centre tracks births, deaths, migration, and other features on a quarterly basis (the Navrongo Demographic Surveillance System, NDSS). Malaria is holoendemic ${ }^{11}$ and is the most important cause of morbidity and mortality. ${ }^{12}$

Study subjects. Two cohorts were recruited, one at the beginning of the dry season in November 1996 and another at the beginning of the wet season in May 1997. Children between the ages of six and 24 months were eligible for participation. They were selected for recruitment by stratification of 


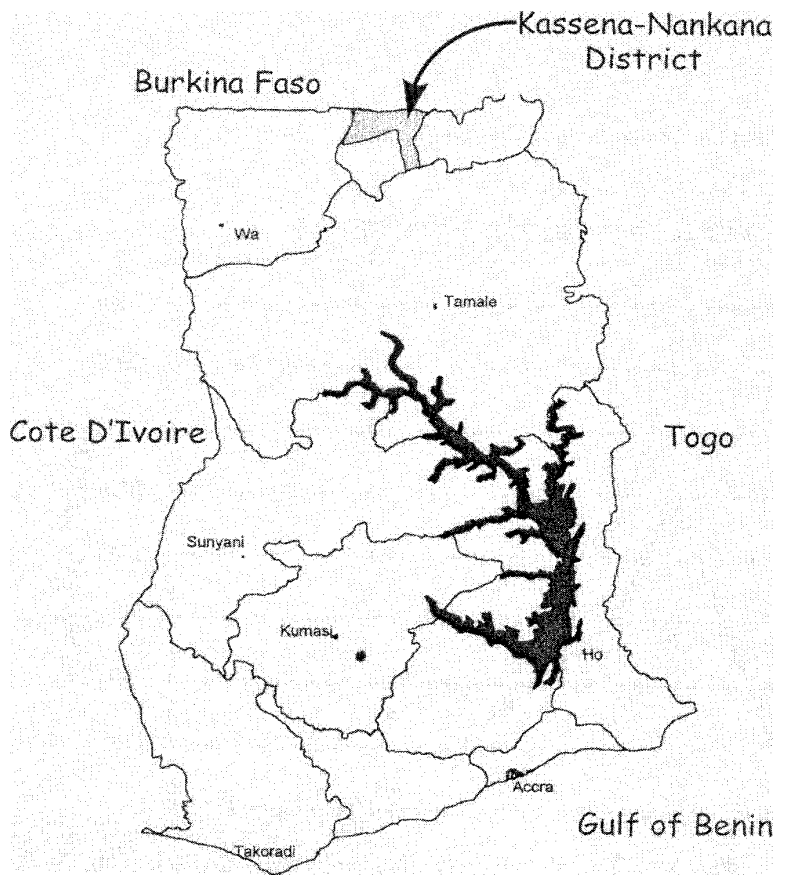

FIGURE 1. Location of the Kassena-Nankana District in northern Ghana.

the study site followed by a randomized selection of 15 index clusters of family compounds among the four geographic regions of the district recognized by the NDSS. The number of subjects recruited from each zone represented a sample weighted according to the proportional contribution to the entire population of the district.

Meetings were held with tribal and family group leaders representing the selected index compounds. The rationale, methods, risks, and benefits of the study were explained and questions were addressed. The assent of these leaders represented a key element of the process of informed consent within the cultural context of northern Ghana. We nonetheless repeated the process with individual volunteers at enrollment in accordance with U.S. Navy regulations concerning the use of human subjects of medical research (SECNAVINST 3900.39B) and those of the Ministry of Health, Republic of Ghana. The verbal explanation and the written informed consent forms were in the native languages of the subjects (Kasem or Nankam).

Each index cluster had a target of 19 study subjects. However, variability in the number of eligible subjects per compound required a uniform strategy for reaching beyond the randomly identified index cluster. When the index compound failed to yield the target of 19 subjects, the closest compound to the east served as a secondary target for recruitment. If necessary, the process was repeated in adjacent compounds to the north, west, and south, always in that order. The population-weighted random selection of index compounds among zones assured all eligible children in the district had an equal probability of selection.

Mothers indicating a willingness to participate in the study were transported with eligible children to an enrollment station. During the two week enrollment phase of each cohort, approximately 40 subjects were screened daily by the research team. Children with peripheral blood hemoglobin concentra-

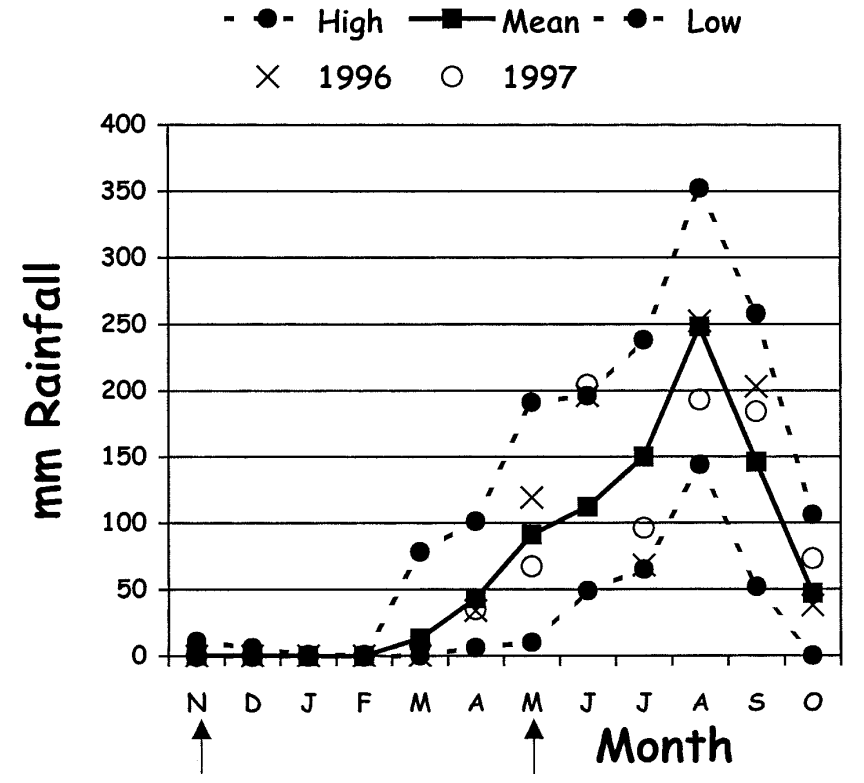

Figure 2. Maximum, minumum (solid dots, dotted lines) or mean (squares, solid line) monthly rainfall in the Kassena-Nankana District in northern Ghana between 1978 and 1995, and monthly rainfall during $1996(\times)$ and $1997(\bigcirc)$. The arrows at November and May indicate enrollments for the dry season and wet season cohorts, respectively.

tions $>5.9 \mathrm{~g} / \mathrm{dl}$ and free of disease requiring hospitalization were enrolled. Among the 349 children screened at the beginning of the dry season (end of wet season), 94 were excluded due to severe anemia. These severely anemic children were taken to the district hospital for treatment. Among the 286 children screened at the beginning of the wet season, 275 were enrolled; only four of 11 exclusions had severe anemia. The report by Koram and others ${ }^{10}$ details the marked differences in prevalence of severe anemia between these two randomly selected cohorts. Table 1 lists the statistical summary of each cohort.

Radical cure. Radical cure started on the day following enrollment. Subjects received quinine sulfate (Cox Pharmaceuticals, Fort Lee, NJ, or Royce Laboratories, Miami, FL; 10 $\mathrm{mg} / \mathrm{kg}$, three times a day for four days), followed by Fansidar ${ }^{\circledR}$ (F. Hoffmann LaRoche Pharmaceuticals, Basel, Switzerland; half a tablet for 5-10 kg of body weight, and one tablet for > $10 \mathrm{~kg}$, single dose). Subjects testing normal for glucose-6phosphate dehydrogenase (G6PD, assay by NADP+ spot test; Sigma Chemical Co., St. Louis, MO) received $0.3 \mathrm{mg}$ of primaquine base per kilogram of body weight (primaquine phos-

TABLE 1

Comparison of dry and wet season cohorts at enrollment of infants and small children in the Kassena-Nankana District of northern Ghana

\begin{tabular}{lccc}
\hline \multicolumn{1}{c}{ Feature* } & Dry season cohort & Wet season cohort & $P$ \\
\hline Sample size & 259 & 277 & \\
Male : Female & 0.9923 & 1.022 & 0.865 \\
Mean age (months) & 14.98 & 14.83 & 0.749 \\
Mean weight (kg) & 7.97 & 7.80 & 0.211 \\
\% G6PD deficient & 6.95 & 9.71 & 0.247 \\
Parasitemia & $69 \%$ & $52 \%$ & 0.001 \\
\hline *G6PD = glucose-6-phosphate dehydrogenase. & &
\end{tabular}


phate; Sanofi Winthrop Pharmaceuticals, New York, NY) once a day for 14 days. A licensed pharmacist prepared all of these medications in flavored syrups. A trained field health worker employed by the research team used a syringe to dispense volumes of medicated syrup prescribed by the team physician. Only six of 536 subjects $(1.1 \%)$ missed two or more of the 27 doses. Eighteen of 259 subjects $(6.9 \%)$ in the dry season cohort and 27 of 277 subjects $(9.7 \%)$ in the wet season cohort tested positive for G6PD deficiency and primaquine was withheld from their radical cure.

Laboratory procedures. We used the qualitative NADP+ spot test with modifications to the procedure recommended by the manufacturer (Sigma Chemical Co.). Batches of 30 specimens were tested using a single positive control spot (G6PD-deficient control; Sigma Chemical Co.). Prescribed mixtures of blood $(10 \mu \mathrm{l})$, buffer, and G6PD substrate were incubated at room temperature (approximately $30^{\circ} \mathrm{C}$ ) for 10 min, blotted onto filter paper, air dried, and read in a darkened room under ultraviolet light. We did not use a $37^{\circ} \mathrm{C}$ water bath or readings at timed intervals as prescribed by the kit manufacturer. Subjects diagnosed as G6PD-deficient produced blots that failed to fluoresce to the same extent as most other blots (i.e., the negative control), and to the same extent as the known G6PD-deficient sample on the same blotter (the positive control).

Hemoglobin levels were measured using the Hemocue ${ }^{\circledR}$ (HemoCue AB, Ängelholm, Sweden) system. A microcuvette preloaded with stable reagents withdrew approximately $10 \mu \mathrm{l}$ of blood from the site of puncture. It was immediately placed into a portable spectrophotometric instrument. Stable control cuvettes provided by the manufacturer confirmed proper operation of the instrument. Axillary temperatures were obtained using digital thermometers placed under the arm.

Thick and thin blood films were stained with Giemsa reagents in the usual manner and were read by an experienced malaria microscopist on the day collected. Two hundred optical fields under $1,000 \times$ oil immersion magnification were examined before a film was recorded as negative. Counts of parasites in the thick film were based on the number of asexual parasites per 200 white blood cells (WBCs). This number was converted to parasites per microliter of whole blood using a conversion multiple of 40 (assumes 8,000 $\mathrm{WBCs} / \mu \mathrm{l})$.

Follow-up. A trained field health worker with at least a secondary school education was assigned to each index compound for the 18 days of radical cure and the 20 -week followup period. The field worker visited each compound within his or her area three times per week to query mothers/guardians of the health of their child. Complaint of illness prompted transport of the mother and child to the district hospital for examination by a physician, including a blood film examination for plasmodia. Every two weeks a blood film was collected whether or not the mother complained of illness in her child. Children with malaria were treated with Lariam ${ }^{\circledR}$ mixed in syrup (mefloquine $\mathrm{HCl}$; F. Hoffmann LaRoche Pharmaceuticals; $20 \mathrm{mg}$ base $/ \mathrm{kg}$ of body weight in a single dose). Whether administered after an unscheduled visit to hospital or after a routine blood film examination, therapy with mefloquine marked the endpoint of participation in this study. The efficacy of mefloquine for therapy in these cohorts was virtually complete (Baird JK and others, unpublished data).
Clinical analysis. Children presenting with signs of disease (fever, anemia) and patent parasitemia were considered to be ill with malaria. Although other diseases certainly occur in the district, and we have not yet estimated the risk attributable to malaria among febrile or anemic children, malaria infection overwhelmingly dominates the burden of disease in these communities. An important exception to this rule is epidemic meningococcal meningitis, which did not occur during these studies. Evidence of disease reported by the mother, including fever, rigors, or vomiting, when coupled with patent parasitemia were attributed to malaria. Although the lower limit for permissible hemoglobin at enrollment was $6.0 \mathrm{~g} / \mathrm{dl}$, we used a hemoglobin level $<8.0 \mathrm{~g} / \mathrm{dl}$ to define anemia as a disease endpoint in the presence of parasitemia.

Epidemiologic analysis. Incidence Density. Incidence density estimates the force of infection, or new infections per unit person-time. The administration of radical cure allowed classification of post-treatment parasitemia as a new infection. In the absence of cure, recrudescence and relapse confounds the estimate. We used the direct method to measure incidence density; the number of post-treatment parasitemias divided by the sum of person-time at risk. Subjects no longer contributed person-time to the denominator after becoming parasitemic or when lost to follow-up.

The incidence density of other endpoints was measured. Parasitemia with fever (axillary temperature $>99^{\circ} \mathrm{F}$ ) or with anemia (hemoglobin $<8.0 \mathrm{~g} / \mathrm{dl}$ ) at parasitemia densities $>$ $5,000 / \mu \mathrm{l},>10,000 / \mu \mathrm{l},>20,000 / \mu \mathrm{l}$, and $>50,000 / \mu l$ constituted these endpoints. Subjects having parasitemia without the specified outcome variable were designated as dropouts in these estimates, i.e., contributing person-time to the denominator up to the time of unqualified parasitemia.

We used incidence densities to calculate the risk of an outcome in one group relative to the risk experienced by another group using SPSS version 8.0 (SPSS, Inc., Chicago, IL). A relative risk of 1.0 reflected no difference in the risk to the two groups, and the distance from 1.0 (in either direction) represented the degree of difference between the rates. We calculated $95 \%$ confidence intervals (CIs) around the relative risks as measures of the precision of our estimates. The significance of the difference between the rates was determined using Fisher's exact test or a chi-square test depending on number of events within groups.

Cumulative incidence. Cumulative incidence expresses the risk of an outcome over a defined period as a proportion. The primary risk evaluated in this study was first parasitemia after radical cure. We also calculated risk of high density parasitemia $(>20,000 / \mu 1)$. The first 10 days following radical cure were considered to be free of risk, and were excluded from the denominator of the estimate of cumulative incidence. The life table method using weekly intervals estimated cumulative incidence. When calculating the risk of developing a parasitemia $>20,000 / \mu l$, subjects developing parasitemias $<20,000 /$ $\mu l$ were treated as withdrawals, i.e., contributing to the denominator up to the interval in which they became parasitemic (assuming a mid-interval event for all subjects).

\section{RESULTS}

Radical cure. The prevalence of parasitemia at enrollment was $69 \%$ and $52 \%$ in the dry and wet season cohorts, and this probably accounted for much of the vomiting that occurred 
TABLE 2

Age-specific geometric mean Plasmodium falciparum parasite densities (95\% confidence interval) in infants and small children in the Kassena-Nankana District in northern Ghana

\begin{tabular}{lrrr}
\hline Age group (months) & Dry season cohort & Wet season cohort & $P$ \\
\hline A. Enrollment & & & \\
$<12$ & $1,515(949-2,418)$ & $925(528-1,620)$ & 0.194 \\
$12-18$ & $811(470-1,401)$ & $1,169(645-2,120)$ & 0.376 \\
$>18$ & $1,084(696-1,688)$ & $1,150(706-1,875)$ & 0.860 \\
$\quad$ All ages & $1,112(842-1,470)$ & $1,099(801-1,507)$ & 0.911 \\
B. Post-Radical Cure & & \\
$\quad<12$ & $3,796(2,161-6,667)$ & $1,704(970-2,991)$ & 0.061 \\
$12-18$ & $2,580(1,461-4,555)$ & $5,131(2,629-10,013)$ & 0.127 \\
$>18$ & $3,564(2,111-6,015)$ & $3,716(2,174-6,353)$ & 0.913 \\
All ages & $3,310(2,409-4,547)$ & $3,056(2,177-4,290)$ & 0.737 \\
\hline
\end{tabular}

during radical cure. Repeated vomiting (three or more episodes) occurred in $4.7 \%$ and $3.6 \%$ of subjects taking quinine in the dry and wet season cohorts, respectively $(P>0.1)$. After the single dose of Fansidar, ${ }^{\circledR} 0.8 \%$ and $6.2 \%$ of subjects reported vomiting in the dry and wet season cohorts $(P<$ $0.001)$. During the administration of primaquine in the dry season and wet season cohorts, $4.6 \%$ and $6.8 \%$ of subjects had repeated vomiting $(P>0.1)$. Despite the risk of vomiting, 531 of 537 subjects $(98.9 \%)$ beginning radical cure completed the regimen, and 530 had negative blood films on day 15 of radical cure (>99\% efficacy).

Point measures. Enrollment. The two cohorts were virtually identical with respect to composition by sex, age, weight, and frequency of G6PD deficiency (Table 1). The prevalence of parasitemia at enrollment was significantly higher in the dry season cohort (end of wet season) than in the wet season cohort (end of dry season); $69 \%$ versus $52 \% ; P<0.0001$. However, there was no difference either overall or within specific age groups in geometric mean parasite densities between wet and dry season cohorts at enrollment (Table 2A).

First Parasitemia. The density of first parasitemia after radical cure was also similar among age groups between the wet and dry seasons (Table 2B). Infants $<12$ months old in the dry season cohort exhibited a mean parasite density after radical cure that approached being significantly higher compared to those in the wet season cohort $(P=0.061$; Table 2B).

The prevalence of fever with first parasitemia was $31 \%$ in the dry season cohort and $34 \%$ in the wet season (relative risk $=0.91,95 \% \mathrm{CI}=0.69-1.18 ; P=0.464)$. Only children $12-18$ months old had a slightly higher risk of fever during the wet season (relative risk $=0.67,95 \% \mathrm{CI}=0.42-1.07 ; P=0.083$ ).

Incidence density. Figure 3 shows the incidence density of infection among index compounds representing the sample evaluated in the wet and dry season cohorts. The data do not include 21 subjects that could not be retrospectively assigned to an index compound. Only 11 of 15 index compounds (A through $\mathrm{K}$ in Figure 3) appear in this analysis because proximate compounds were lumped together due to uncertainty with original index assignment. All 15 index compounds were represented among the 11 clustered compounds shown in Figure 3. Index compounds in the wet season cohort had incidence densities ranging from 6.42 to 10.4 per person-year $(P$ $>0.10$, by the Mantel-Cox log rank test). Index compounds in the dry season cohort had incidence densities ranging from 1.12 to 7.29 per person-year $(P>0.10)$. These data illustrate the relatively uniform incidence of new infection among the

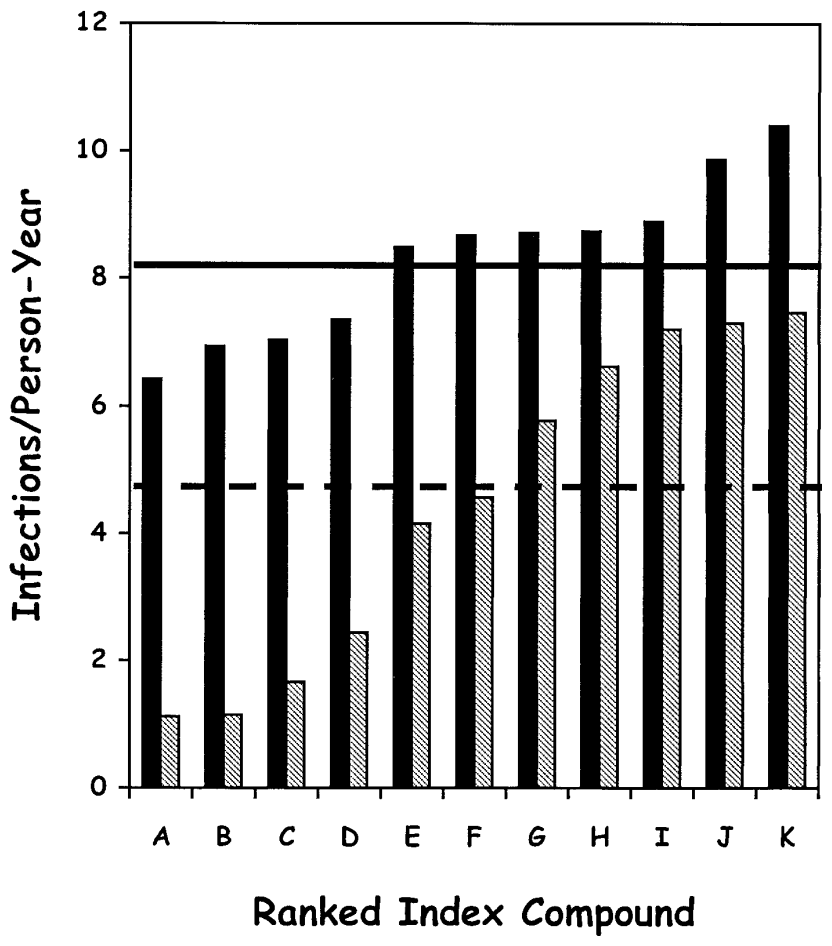

FIGURE 3. Incidence density among index compounds representing the sample population for the wet (solid bars) and dry (dashed bars) season cohorts. Variance from the mean incidence density in the wet season (solid line) and dry season (dashed line) among index compounds was not significant $(P>0.10$ and $P>0.10)$ by the MantelCox log rank test.

randomly selected index compounds in both the wet and dry season cohorts.

Table 3 lists the incidence densities of new infection in the wet and dry season cohorts. The table stratifies incidence density according to parasite density and accompanying illnesses, i.e., with fever or anemia. Incidence density of any parasitemia in the wet season was significantly higher than in the dry season; 7.11 versus 4.71 infections/person-year (relative risk $=1.51,95 \% \mathrm{CI}=1.25-1.81 ; P=0.00001)$. The relative risk was still higher when fever was included in the endpoint; 1.46 versus 2.43 infections/person-year (relative risk $=1.66,95 \%$ CI $=1.20-2.31 ; P=0.0023$ ). However, for parasitemia with anemia, there was no difference in incidence density between wet and dry season cohorts (relative risk = $1.02, P=0.9915)$.

The pattern of significant differences in incidence density between any parasitemia, or those with fever but not anemia in the wet and dry season cohorts, appeared at all strata of parasite density (Table 3 ). In general, the greater the parasite density, the greater the relative risk for that outcome in the wet season cohort. Compared with the dry season, the risk of having a febrile parasitemia of any density was 1.66 times higher than that in the wet season. Similarly, the risk of developing febrile parasitemia $>5,000 / \mu \mathrm{l},>10,000 / \mu \mathrm{l},>$ $20,000 / \mu \mathrm{l}$, and $>50,000 / \mu \mathrm{l}$ during the wet season was 1.92 , $1.93,2.45$, and 4.33 times that in the dry season respectively. The $95 \%$ CIs for all of these relative risks did not include 1.0, and the $P$ values were always $<0.0025$. In contrast, the relative risk of parasitemia with anemia never produced $95 \%$ CIs that excluded 1.0, and the $P$ values were never $<0.33$. 
TABLE 3

Incidence density of Plasmodium falciparum parasitemia post-radical cure in infants and small children in the Kassena-Nankana District of northern Ghana

\begin{tabular}{|c|c|c|c|c|c|c|c|}
\hline \multirow[b]{2}{*}{ Endpoint } & \multicolumn{2}{|c|}{ Dry season } & \multicolumn{2}{|c|}{ Wet season } & \multirow[b]{2}{*}{$\begin{array}{c}\text { Relative } \\
\text { risk } \dagger\end{array}$} & \multirow[b]{2}{*}{$95 \% \mathrm{CI} \ddagger$} & \multirow[b]{2}{*}{$P \S$} \\
\hline & $\mathrm{n}$ & $\begin{array}{c}\begin{array}{c}\text { Incidence } \\
\text { density* }\end{array} \\
\end{array}$ & $\mathrm{n}$ & $\begin{array}{l}\text { Incidence } \\
\text { density* }\end{array}$ & & & \\
\hline \multicolumn{8}{|l|}{ Any parasitemia } \\
\hline \pm fever, anemia & 200 & 4.71 & 254 & 7.11 & 1.51 & $1.25-1.81$ & 0.00001 \\
\hline With feverII & 62 & 1.46 & 87 & 2.43 & 1.66 & $1.20-2.31$ & 0.00225 \\
\hline With anemia\# & 73 & 1.72 & 63 & 1.76 & 1.02 & $0.73-1.44$ & 0.99147 \\
\hline \multicolumn{8}{|c|}{ Parasitemia $>5,000 / \mu l$} \\
\hline \pm fever, anemia & 90 & 2.18 & 121 & 3.39 & 1.56 & $1.18-2.04$ & 0.00146 \\
\hline With feverII & 37 & 0.89 & 61 & 1.71 & 1.92 & $1.27-2.87$ & 0.00221 \\
\hline With anemia\# & 33 & 0.80 & 27 & 0.76 & 0.95 & $0.57-1.58$ & 0.89733 \\
\hline \multicolumn{8}{|c|}{ Parasitemia $>10,000 / \mu l$} \\
\hline \pm fever, anemia & 78 & 1.89 & 103 & 2.88 & 1.53 & $1.14-2.05$ & 0.00458 \\
\hline With feverII & 33 & 0.80 & 55 & 1.54 & 1.93 & $1.25-2.97$ & 0.00264 \\
\hline With anemia\# & 30 & 0.73 & 25 & 0.70 & 0.96 & $0.57-1.64$ & 1.00000 \\
\hline \multicolumn{8}{|c|}{ Parasitemia $>20,000 / \mu l$} \\
\hline \pm fever, anemia & 55 & 1.33 & 81 & 2.27 & 1.71 & $1.21-2.40$ & 0.00249 \\
\hline With feverII & 23 & 0.56 & 49 & 1.37 & 2.45 & $1.50-4.05$ & 0.00022 \\
\hline With anemia\# & 20 & 0.48 & 18 & 0.50 & 1.04 & $0.55-1.97$ & 1.00000 \\
\hline \multicolumn{8}{|c|}{ Parasitemia $>50,000 / \mu l$} \\
\hline \pm fever, anemia & 18 & 0.44 & 53 & 1.48 & 3.36 & $2.00-5.82$ & 0.00000 \\
\hline With feverII & 10 & 0.24 & 37 & 1.04 & 4.43 & $2.13-8.61$ & 0.00001 \\
\hline With anemia\# & 7 & 0.17 & 10 & 0.28 & 1.65 & $0.63-4.35$ & 0.33789 \\
\hline
\end{tabular}

* Outcomes per person-year.

$\dagger$ The ratio of wet season rates over dry season.

$\doteqdot$ The confidence intervals (CIs) are Sato limits.

\$The $P$ value is Fisher's exact (two-tail) chi-square.

\# Anemia is defined as a hemoglobin level $<8 \mathrm{~g} / \mathrm{dl}$.

Cumulative incidence. Figure 4 shows the cumulative incidence of all parasitemias and parasitemias $>20,000 / \mu$ l in the two cohorts. The cumulative incidence of parasitemia after radical cure increased more slowly during the first three

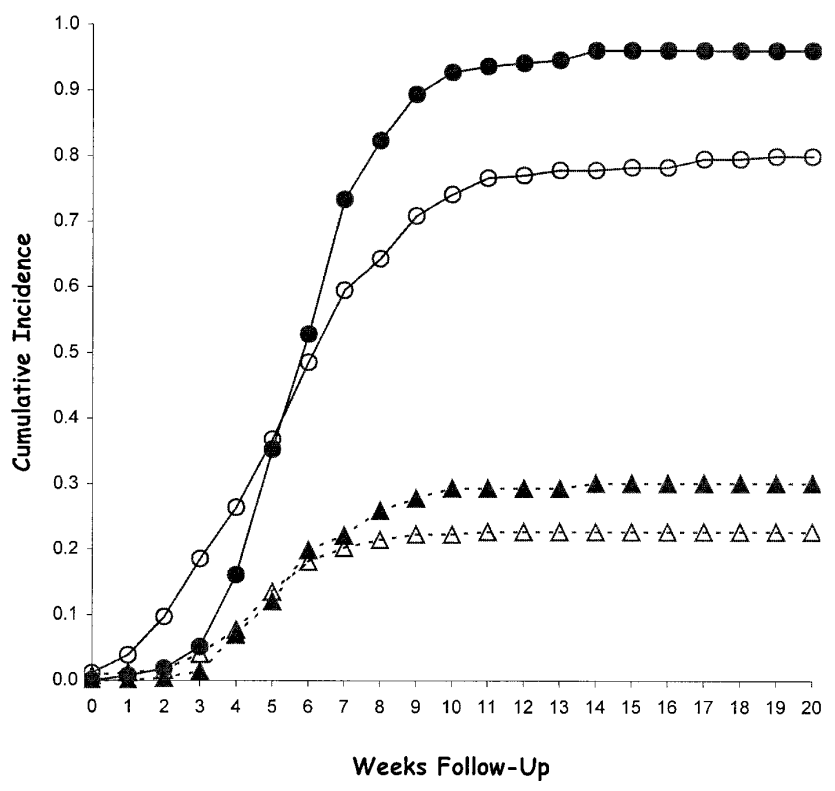

Figure 4. Cumulative incidence of any parasitemia by Plasmodium falciparum in infants and small children during the wet (solid circles) and dry (hollow circles) seasons, along with cumulative incidence of high density parasitemia $(>20,000 / \mu \mathrm{l})$ in the wet (solid triangles) and dry (hollow triangles) seasons in the Kassena-Nankana District of northern Ghana. weeks of risk in the wet season cohort compared with the same period in the dry season cohort (cumulative incidence $=0.19$ versus $0.04 ; P=0.0025$ ). The 20 -week cumulative incidence of parasitemia in the dry season cohort reached a plateau at 0.76 at the end of 12 weeks, leaving about onequarter of the cohort virtually risk-free for the remaining two months of follow-up. In contrast, the wet season cohort reached a plateau cumulative incidence of 0.93 at the end of 10 weeks. During the remaining 10 weeks of presumably high risk, only an additional $3 \%$ of subjects became infected. A total of six subjects remained free of infection throughout the study, and these accounted for the $3.5 \%$ risk of not being infected. The 20 -week cumulative incidence of infection by $P$. falciparum was $96.5 \%$. The risk of parasitemia $<20,000 / \mu 1$ peaked at approximately $25 \%$ in both cohorts at about the same time, at the end of nine weeks.

\section{DISCUSSION}

Intense transmission of $P$. falciparum occurred from May to February in the Kassena-Nankana District of northern Ghana. The incidence density of infection decreased moderately during the dry season that commenced in October. Transmission abruptly dropped to very low levels only during the last two to three months of the dry season (Figure 4). No significant differences appeared among children of any age in the dry and wet season cohorts with respect to geometric mean density of parasitemia at either enrollment or first parasitemia (Table 2A and B). Appreciable differences between seasons appeared only when the data were stratified by parasite count and fever (Table 3 ). The relative risk of parasitemia with fever at any density, $>5,000 / \mu l,>10,000 / \mu l,>20,000 / \mu l$, 
and $>50,000 / \mu$ for the wet season cohort relative to the dry season cohort was $1.66,1.92,1.89,2.45$, and 4.33 , respectively. Risk of parasitemia with fever increased in proportion to the density of parasitemia during the wet season. In both cohorts, the incidence density of these parasitemia outcomes diminished sharply with increasing counts. The outcomes of greatest relative risk during the wet season represented low frequency events, e.g., parasitemia $>50,000 / \mu$ l with fever was four times more likely to occur during the wet season, but occurred less frequently than the lower parasitemia levels with fever (1.04 versus 1.71 events per person-year).

Despite the relatively subtle parasitologic and clinical distinctions between the dry and wet season cohorts, the statistical power of vaccine trials applying endpoints of high parasitemia and fever would be enhanced by execution framed to capture wet season risks. The example of risk of parasitemia $>50,000 / \mu \mathrm{l}$ with fever measured in these studies best illustrates this point. If one assumes a vaccine efficacy of $85 \%$, and the operational requirement to have an $80 \%$ probability of having a $95 \%$ CI that excludes $70 \%$ (i.e., the lower limit of acceptability for efficacy), then the dry season incidence rate of 0.24 events per person year for this outcome demands a sample size of 2,055. Applying the same parameters to the 1.04 events per person year incidence rate in the wet season gives a sample size estimate of just 363 subjects. Relatively subtle distinctions in seasonal end point incidence densities may profoundly impact sample size requirements in intervention trials.

Anemia is an important feature of morbidity caused by malaria in the Kassena-Nankana District. The infants and young children in these studies showed dramatic distinctions with respect to the prevalence of severe anemia as seen at enrollment at the end of the dry and wet seasons. ${ }^{10}$ In effect, an epidemic of severe malarial anemia occurred in these children as the wet season progressed: apparently starting at $1 \%$ in May and increasing to $24 \%$ in October. Given the fact that we have now documented that intense transmission continues at least to February, the prevalence of severe anemia may even worsen relative to the $25 \%$ rate measured in October. The relatively brief respite in transmission between February and May appeared sufficient to allow recovery of most severe anemia to above the $6.0 \mathrm{~g} / \mathrm{dl}$ level. The work reported here and by Koram and others ${ }^{10}$ demonstrates the need for longitudinal studies of seasonal risk of severe anemia in northern Ghana.

The relatively low risk of anemia among our subjects with first parasitemia, and the absence of any differences between the dry and wet season risks of anemia is a product of the experimental design. Chronic infection causes anemia in malaria. ${ }^{13,14}$ Measuring the incidence density of infection necessitated radical cure and, thus, the interruption of chronic parasitemia. Moreover, we could not enroll the $25 \%$ of screened children with severe anemia, so that children in the dry and wet season cohorts began radical cure with similar anemia profiles, parasitologic features, and chemotherapy. Thus, it was not surprising that the first parasitemia of the dry season had no less risk of anemia compared with that in the wet season. This study has demonstrated that intervention trials applying an endpoint that includes severe anemia should allow for a chronic course of parasitemia.

The failure of $2.2 \%$ of the subjects in the wet season cohort to develop parasitemia (accounting for a $3.5 \%$ residual risk) raises potentially important questions. Given the 10-week plateau in the face of intense transmission, it is difficult to assign these residual aparasitemic subjects to chance. These children were somehow protected. Unknown genetic factors may have precluded parasitemia in these children, e.g. HLA or cytokine promoter alleles. ${ }^{15,16}$ However, in an adult cohort from the same gene pools and treated and followed through the wet season in the same manner, ${ }^{17}$ all developed parasitemia. Possible explanations for the minority of child subjects failing to develop parasitemia include consistently applied and extraordinarily effective protective measures by the family (e.g., mosquito netting, repellants, or antimalarials), and genetic or immunogenetic factors that protect children but not adults from parasitemia.

The geometric mean parasitemia densities at enrollment were significantly lower than at first parasitemia post-radical cure. At enrollment, the dry and wet season cohorts had geometric mean densities of 1,112 and $1,099 / \mu 1$, whereas these densities were 3,310 and $3,056 / \mu 1$ at first parasitemia $(P<$ 0.001 for each). Many explanations for these differences are possible, but we consider the effect of radical cure as most likely. Although it is difficult to rule out drug-mediated interference with immune effectors that limit parasite densities as an explanation, we instead favor diminished effectiveness of premunition as a consequence of eradication of parasitemia by radical curative therapy. Premunition is the protection from heavy parasite burdens imbued by lesser parasite burdens. We think the elimination of chronic, relatively lowgrade parasitemia may have established susceptibility to acute, relatively high-grade parasitemia. This carries important implications with regard to experimental design in intervention studies and to control strategies aimed at delivering effective therapies in holoendemic Africa. Studies designed to address this important question should be carried out.

In summary, these studies have documented intense malaria transmission with an incidence density between five and seven infections/person-year through most of the year among children 6-24 months old living in the Kassena-Nankana District of northern Ghana. A period of 2-3 months at the end of the dry season had very low risk of infection, and marked a dramatic decrease in the prevalence of severe anemia. Postradical cure infections carried risk of relatively high-density parasitemia during dry or wet seasons.

Acknowledgments: We express our thanks to the Kassena-Nankana community, especially the volunteers in this study. We also acknowledge the team of health workers attached to the MARS (Malaria Attack Rate Study) project at the Navrongo Health Research Centre; their excellent work made this report possible. In particular, we acknowledge Charles Attiogbe (Noguchi Medical Research Institute) for his input as the study microscopist, and Cletus Tindana, Salifu Abdul Rahman, and Paulina Tindana as field supervisors. Special thanks goes to Drs. Kweku Enos and Mensah-Afful, the study physicians, and especially Dr. Alex Nazzar for essential support and advice. J. Kevin Baird and Gregory C. Utz gratefully acknowledge the support and assistance of Troy Fitrell, Vice Consul at the American Embassy in Accra, Ghana.

Disclaimer: The views of the authors are their own and do not purport to reflect those of the U.S. Navy or the Department of Defense. This work was supported by an Independent Research initiative granted to GU by the Naval Medical Research and Development Command, Bethesda, Maryland.

Authors' addresses: J. Kevin Baird and Mazie J. Barcus, Parasitic Diseases Program, U.S. Naval Medical Research Unit \#2, American Embassy, Jakarta, FPO AP 96520-8132 USA. Owusu Agyei and 
Fred N. Binka, Navrongo Health Research Centre, Navrongo, Ghana. Trevor R. Jones, David J. Fryauff, and Stephen L. Hoffman, Naval Medical Research Center, Silver Spring, MD. Gregory C. Utz, U.S. Naval Medical Research Unit No. 3, Cairo, Egypt. Kwadwo Koram and Francis N. Nkrumah, Noguchi Memorial Institute for Medical Research, Accra, Ghana.

Reprint requests: J. Kevin Baird, NAMRU-2, American Embassy Jakarta, FPO AP 96520 USA.

\section{REFERENCES}

1. Pull JH, Grab B, 1974. A simple epidemiologic model for evaluating the malaria inoculation rate and the risk of infection in infants. Bull World Health Organ 51: 507-516.

2. Beier JC, Oster CN, Onyango FK, Bales JD, Sherwood JA, Perkins PV, Chumo DK, Koech DK, Whitmire RE, Roberts DR, Diggs CL, Hoffman SL, 1994. Plasmodium falciparum incidence relative to entomologic inoculation rates at a site proposed for testing malaria vaccines in western Kenya. Am $J$ Trop Med Hyg 50: 529-536.

3. McElroy PD, Beier JC, Oster CN, Onyango FK, Oloo AJ, Lin X. Beadle C, Hoffman SL, 1997. Dose- and time-dependent relations between infective Anopheles inoculation and outcomes of Plasmodium falciparum parasitemia among children in Western Kenya. Am J Epidemiol 145: 945-956.

4. Pan American Health Organization, 1997. Status of Malaria Programs in the Americas. XLV Report. Washington, DC.

5. Delacollette C, van der Stuyft P, 1993. High parasitemia incidence rates can be used to estimate malaria morbidity rates. Ann Trop Med Parasitol 87: 537-539.

6. Snow RW, Omumbo JA, Lowe B, Molyneux CS, Obiero JO, Palmer A, Weber MW, Pinder M, Nahlen B, Obonyo C, Newbold C, Gupta S, Marsh K, 1997. Relation between severe malaria morbidity in children and level of Plasmodium falciparum transmission in Africa. Lancet 349: 1650-1654.
7. Alles HK, Mendis KN, Carter R, 1998. Malaria mortality rates in South Asia and in Africa: implications for malaria control. Parasitol Today 14: 369-375.

8. Gupta S, Snow RW, Donnelly CA, Marsh K, Newbold C, 1999. Immunity to non-cerebral malaria is acquired after on or two infections. Nature Med 5: 340-343.

9. Modiano D, Sirima BS, Sawadogo A, Sanou I, Pare J, Konate A, Pagnoni F, 1998. Severe malaria in Burkina Faso: influence of age and transmission level on clinical presentation. Am J Trop Med Hyg 59: 539-542.

10. Koram KA, Owusu-Agyei S, Utz G, Binka FN, Baird JK, Hoffman SL, Nkrumah FK, 2000. Severe anemia in young children after high and low malaria transmission seasons in the Kassena Nankana District of northern Ghana. Am J Trop Med Hyg 62: 670-674.

11. Binka FN, Morris SS, Ross DA, Arthur P, Aryeetey ME, 1994. Patterns of malaria morbidity and mortality in northern Ghana. Trans R Soc Trop Med Hyg 88: 381-385.

12. Binka FN, Maude GH, Gyapong M, Ross DA, Smith PG, 1995. Risk factors for child mortality in northern Ghana: a casecontrol study. Int J Epidemiol 24: 127-135.

13. Miller LH, Good MF, Milon G, 1994. Malaria pathogenesis. Science 264: 1878-1883.

14. Newton CR, Taylor TE, Whitten RO, 1998. Pathophysiology of fatal falciparum malaria in African children. Am J Trop Med Hyg 58: 673-683.

15. Hill AVS, 1999. The immunogenetics of resistance to malaria. Proc Assoc Am Physicians 111: 272-277.

16. McGuire W, Knight JC, Hill AVS, Allsopp CE, Greenwood BM, Kwiatkowski D, 1999. Severe malarial anemia and cerebral malaria are associated with different tumor necrosis factor promoter alleles. J Infect Dis 179: 287-290.

17. Owusu-Agyei S, Koram KA, Baird JK, Utz GC, Binka FN, Nkrumah FK, Fryauff DJ, Hoffman SL, 2001. Incidence of symptomatic and asytmptomatic Plasmodium falciparum infection following curative therapy in adult residents of northern Ghana. Am J Trop Med Hyg 65: 197-203. 